\title{
Chest Breadths to Predict Individuals' Age A Case Based View
}

\author{
Andréa Domingues \\ Department of Informatics, University of Minho \\ Braga, Portugal \\ andrea.domingues.1993@gmail.com \\ Henrique Vicente \\ Departamento de Química, Escola de Ciências e \\ Tecnologia, Universidade de Évora, Évora, Portugal \\ Algoritmi Center, University of Minho \\ Braga, Portugal \\ hvicente@uevora.pt
}

\begin{abstract}
It is well known that rib cage dimensions depend on the gender and vary with the age of the individual. Under this setting it is therefore possible to assume that a computational approach to the problem may be thought out and, consequently, this work will focus on the development of an Artificial Intelligence grounded decision support system to predict individual's age, based on such measurements. On the one hand, using some basic image processing techniques it were extracted such descriptions from chest X-rays (i.e., its maximum width and height). On the other hand, the computational framework was built on top of a Logic Programming Case Base approach to knowledge representation and reasoning, which caters for the handling of incomplete, unknown, or even contradictory information. Furthermore, clustering methods based on similarity analysis among cases were used to distinguish and aggregate collections of historical data in order to reduce the search space, therefore enhancing the cases retrieval and the overall computational process. The accuracy of the proposed model is satisfactory, close to $90 \%$.

Keywords - Intelligent Systems; Chest X-ray Images; Logic Programming; Knowledge Representation and Case Based Reasoning.
\end{abstract}

\author{
João Neves \\ Drs. Nicolas \& Asp \\ Dubai, United Arab Emirates \\ joaocpneves@gmail.com \\ Victor Alves, José Neves* \\ Algoritmi Center, University of Minho \\ Braga, Portugal \\ \{valves, jneves\}@di.uminho.pt \\ * Corresponding author: phone: +351-934201337; fax: \\ +351-253604471; e-mail: jneves@di.uminho.pt
}

